\title{
The Influence of "Likes" on User Content Generation in Online Investment Communities
}

\author{
Xiaolin Zhuo \\ School of Management, \\ Harbin Institute of \\ Technology \\ hitzhuoxl@126.com
}

\author{
Hong Hong \\ School of Management, \\ Harbin Institute of \\ Technology \\ honghong@hit.edu.cn
}

\author{
Dapeng Xu \\ School of Management, \\ Harbin Institute of \\ Technology \\ dpxu@hit.edu.cn
}

\author{
Qiang Ye \\ School of Management, \\ Harbin Institute of \\ Technology \\ yeqiang@hit.edu.cn
}

\begin{abstract}
Investors increasingly rely on investment advice in online investment communities (OICs). This study analyzes the influence of the "likes" function on the content generation in OICs. Based on the data collected from Seeking Alpha, we perform a series of analyses from the perspectives of both authors and readers. From the angle of authors, we find that authors express the logic of the articles more seriously by increasing the use of negative words, and reducing the frequency of writing articles. The reader-level analyses show that "likes" and "comments" are complementary to each other, and readers do not reduce their "comments" after the introduction of the "likes" function. In general, the launch of the new function affects the content generated by both authors and readers. Our study can enrich the research on user-generated content (UGC) and provide helpful suggestions to OIC managers in motivating users to make feedbacks and contributions in such communities.
\end{abstract}

\section{Introduction}

Driven by the development and opening of the Internet, Internet users are more and more willing to share and spread contents they generated on the network, and user-generated content (UGC) community has evolved from one-side platforms of releasing information to users to social media. The field of financial investment is also quietly changing relying on UGC. Users can share investment ideas through Online investment communities (OICs) such as Seeking Alpha and interact within the community. Different from other topic forum, financial investments are professional and involve monetary returns, a rigorous, professional OIC is important to investors. In recent years, OICs have reduced the information asymmetry in the investment market to a certain extent [1]. Many scholars have paid attention to this phenomenon. They find that investor sentiment in OICs is strongly related to stock price, and users in OIC indeed provide valuable investment advice to investors [2-6]. The popularity of OICs is of significant value to investment research, but most of the research focuses on how investment communities affect the stock market, while the research on the content of OICs is rare.

The sustainable development of OICs depends on how to motivate users to generate content actively. Previous studies have shown that making voluntary contributions to UGC is mainly driven by psychological satisfaction and personal interest $[7,8]$, and positive feedbacks provide the feeling of satisfaction for content contributors. Ding et al. (2017) indicate that "likes" encourages individuals to make repeated contributions to UGCs with higher psychological encouragement. Contributors on Facebook believe that communication can be achieved through clicking the "likes" button, and "likes" stimulate them to update their Facebook [10]. However, the impacts of the "likes" function on UGC in OICs are undiscovered, and the research on the relationship between "likes" and "comments" is very few. The UGC in OICs is mainly composed of investment analysis published by authors and comments written by readers. Therefore, to fill this gap, we seek to address the following research questions: 1) how does the launch of the "likes" function impact the quantity and characteristics of author-generated content in OICs; 2 ) how does the launch of the "likes" function impact the quantity and characteristics of reader-generated content in OICs and what is the relationship between "likes" and "comments".

We utilize Seeking Alpha, one of the largest OICs in America, as the data source to explore the abovementioned research questions. First, our author-level analyses explore how authors change the quantity and content of their articles after the launch of the "likes" function. Second, our reader-level analyses examine how the quantity and emotion of readers' comments change with the introduction of the "likes", and explore the relationship between "likes" and "comments". 
Our results show that authors publish fewer articles after the launch of "likes" function. Besides, in order to obtain more "likes", authors provide more reasoning and logical thinking expressions and use more negative emotions after the implement of "likes" function. In the reader-level analyses, we find that readers write comments more frequently, and their negative emotions are not improved significantly, which means that readers do not give up positive comments because of the new "likes" function. The findings confirm that the "likes" function promotes the generation of comments.

Our study has three main contributions. First, different from other studies focusing on the relationship between investor sentiment and abnormal returns, we discuss the impact of the "likes" function on users' content generation behavior. Our study enriches the literature on OICs and feedback mechanisms. Second, based on previous research, this study explores the specific relationship between "likes" and "comments", and we find the mutual promotion relation of the two feedback methods in OICs. Third, our findings can provide OIC platform managers practical implications for the design of OIC feedback mechanisms.

Our research structure is as follows. In the next section, we describe the theoretical background and put forward our hypotheses. The third section shows our research methodology, which includes data collection, variable measurements, and the establishment of research models. In the fourth section, we perform empirical analyses at both the author-level and the reader-level. The final section is the summary of our contributions, limitations, and future directions.

\section{Theoretical Background and Hypotheses Development}

\subsection{Theoretical Background}

Online communities are kind of public product. Their sustainable development mainly depends on users' active participation and content contributions. Many scholars have studied why users are willing to make voluntary contributions to online communities $[5,11,12]$. Among these reasons, impure altruism provides a satisfactory explanation. Through generating content for communities, contributors get not only utility from the overall supply of public goods but also some private benefits, such as moral satisfaction and the joy of having a lingering fragrance [8].

Given the usefulness of online communities is determined by the quantity and quality of user-generated content, many platforms introduce diverse incentives to motivate user engagement behaviors. For example, some communities provide monetary rewards to contributors who complete a specific task to promote the sharing of valuable information $[13,14]$. Seeking alpha has similar reward mechanism: the author gets $\$ 10$ for every 1,000 views per article. However, according to Chen's research, authors in Seeking Alpha earn only $\$ 33$ a month on average for writing analysis articles[14], this amount clearly does not support or subsidize the author's life. Monetary rewards are not the main reason writers share their ideas. At the same time, other rewards such as "medals" and "likes" also play an important role in online communities. These nonmonetary rewards can increase users' participation and stimulate their creative enthusiasm, thereby making more high-quality contributions $[7,15]$.

"Likes", one of the most common non-monetary incentives, exists in many online communities. "Likes" is a kind of feedback from users who have consumed content. It allows users to express their interest in and support for content by clicking the "likes" button. In a social situation, "likes" also represents a powerful endorsement of and encouragement to contributors who generate the content.

\subsection{Hypotheses Development}

2.2.1. "Likes" Function and Author GeneratedContent. Social effects is the main source of UGC [8]. Participating in online activities (for example, sharing information) can generate psychological encouragement, such as reputation, self-improvement, and social capital, which may affect users' intrinsic motivation $[9,16]$. In the context of OICs, psychological encouragement is also the main motivation for authors to generate content, thereby obtaining approval and a sense of achievement.

As "likes" represents readers' interest in the content and a powerful endorsement of authors, it brings psychological encouragement to authors. As a result, "likes" has encouragement effects on authors" engagement behaviors. Facing the introduction of the "likes" function, the authors may increase their content contributions and publish more articles.

However, the quality of articles in OICs is also important for authors, because the high-quality stock analysis not only helps them retain fans but also brings more attention. The "likes" function provides a new way of reflecting the quality of articles. As a result, the authors may place more weight on quality improvement than quantity increase after the launch of the "likes" function. Generally, the high-quality analysis requires more time and effort, which leads to fewer articles. Therefore, we propose the following two competing hypotheses:

H1a: After the launch of the "likes" function, authors write more articles. 
Hlb: After the launch of the "likes" function, authors write less articles.

Psychological experiments show that individuals are more sensitive to negative information, and they react to negative words more quickly. Negative events leave a more profound impression than positive ones [17]. According to evolutionary psychology, the cost of ignoring negative information is much higher than that of ignoring positive information, because paying attention to negative information is conducive to helping individuals avoid risks [18]. Especially in the financial market, the disclosure of negative news leads to more serious consequences. As a result, market participants show a strong preference for negative opinions to reduce their losses. Accordingly, readers in OICs pay more attention to articles that contain more negative views.

Negative preference in the human brain is reflected in two levels: the reaction to negative information and who publishes negative information [19]. The "likes" function provides readers with a new way to react to articles and authors, strengthening the manifestation of readers' negative preferences. Therefore, after the launch of the "likes" function, authors publish articles with more negative opinions to attract more attention and cater to readers. Meanwhile, to receive more approval, authors would analyze stock value in more detail and integrate more personal analysis into articles. As a result, the articles contain more words that show cognitive processes. Therefore, we put forward hypotheses 2 and 3.

H2: After the launch of "likes" function, authors express more negative emotion in their articles.

H3: After the launch of "likes" function, authors use more cognitive words in their articles.

\subsection{2. "Likes" Function and Reader-Generated}

Content. Using information to make decisions is the basis of business. However, decision makers would be limited by many resources, such as limited funds, time, energy [20] or cognitive ability [21]. Individual investors often cannot bring financial information into trading decisions, but tend to trade on eye-catching trends and events [22]. As a professional information intermediary, analysts can mine more internal information and conduct professional interpretation. Hence, they can affect investors' judgment of the firms' stock price [23]. This is a good explanation for readers' dependence on OICs.

In OICs, "comments" and "likes" are two main ways for readers to interact with authors. However, there is a significant difference between their information processing costs according to information cost theory. Since "likes" only represents readers' endorsement of the content and needs no deep insight, its information processing cost is relatively low [24]. On the contrary, comments contain readers' opinions and evaluations. Generally, they show three attitudes of readers: agreement, supplement, and disagreement. To show their agreement, readers usually write "nutritious" comments with simple praise: "Well done", "Agree!", etc. If OICs introduce the "likes" function, readers could turn to click the "likes" button instead of writing nutritious comments due to the lower information processing cost of "likes". As a result, readers would write comments only when they hold different opinions or hope to supplement authors' views. Therefore, the comments would show higher negative emotions and Hypothesis 4 is proposed.

H4: After the launch of "likes" function, readers' emotions expressed in comments become more negative.

Comments have always been an indispensable interactive design in online communities. As a simple interactive function, "likes" can only express positive emotions. The relationship between "likes" and "comments" is rarely investigated by researchers. As indicated in Hypothesis 4, the lower information cost enables "likes" to replace some praise comments. However, according to user participation theory, the new feedback can enhance the users' identity and provide readers with a novel interaction mode [25]. Enthusiastic responses can bring psychological encouragement for users, which improves users' participation [26]. Therefore, the launch of the "likes" function helps communities improve the enthusiasm of user participation. As a result, many users may write comments after clicking the "likes" button. That is, "likes" and "comment" present a mutually reinforcing relationship rather than a substitution one. Based on the above analyses, we propose the following two competing hypotheses:

H5a: After the launch of "likes" function, readers write less comments.

H5b: After the launch of "likes" function, readers write more comments.

\section{Methodology}

\subsection{Data}

Our platform of interest is Seeking Alpha (https://seekingalpha.com, SA). SA is one of the most popular sources of financial news and stock analysis. By 2020, on average, 20 million users visit Seeking Alpha every month, and more than 7000 contributors publish more than 10000 investment analyses. Seeking Alpha is a typical OIC with two groups of contributors: the first one is authors who generate content by publishing articles; the other one is readers who contribute content by giving feedbacks for articles they read. To motivate two groups of contributors to generate content, SA 
launched a new feedback function, "likes", recently. After the launch of the "likes" function, in addition to writing comments, readers can choose to click the "likes" button after browsing the article. It's worth noting that readers' page views and "likes" are two statistics. "Likes" is a subjective action, expressing support and love for the content. When readers agree with the author's opinion, or think that the author of the effort, the reader will choose to click "like". The author will receive a notification of readers' "likes" and know the total number of "likes". Although there is no official notice to indicate when this function is released, we find that the proportion of liked articles suddenly increased significantly in the 10th and 11th week of 2018 and then tended to be flat according to the history data. An average of $80 \%$ of articles were liked after the 11th week, and this figure has remained at around $80 \%$, indicating that readers quickly perceived the addition of "like". Therefore, we consider the 10th and 11th week of 2018 as the time period for event occurrence and user perception. To examine the effects of the "likes" button, we focus on the changes of user-generated content characteristics in the 8 weeks before the 10th week of 2018 and the 8 weeks after the 11 th week of 2018. As users quickly perceive the new function, a short-time window may avoid mixed impacts brought by other events. Figure 1 shows the percentage of articles that were liked each week.

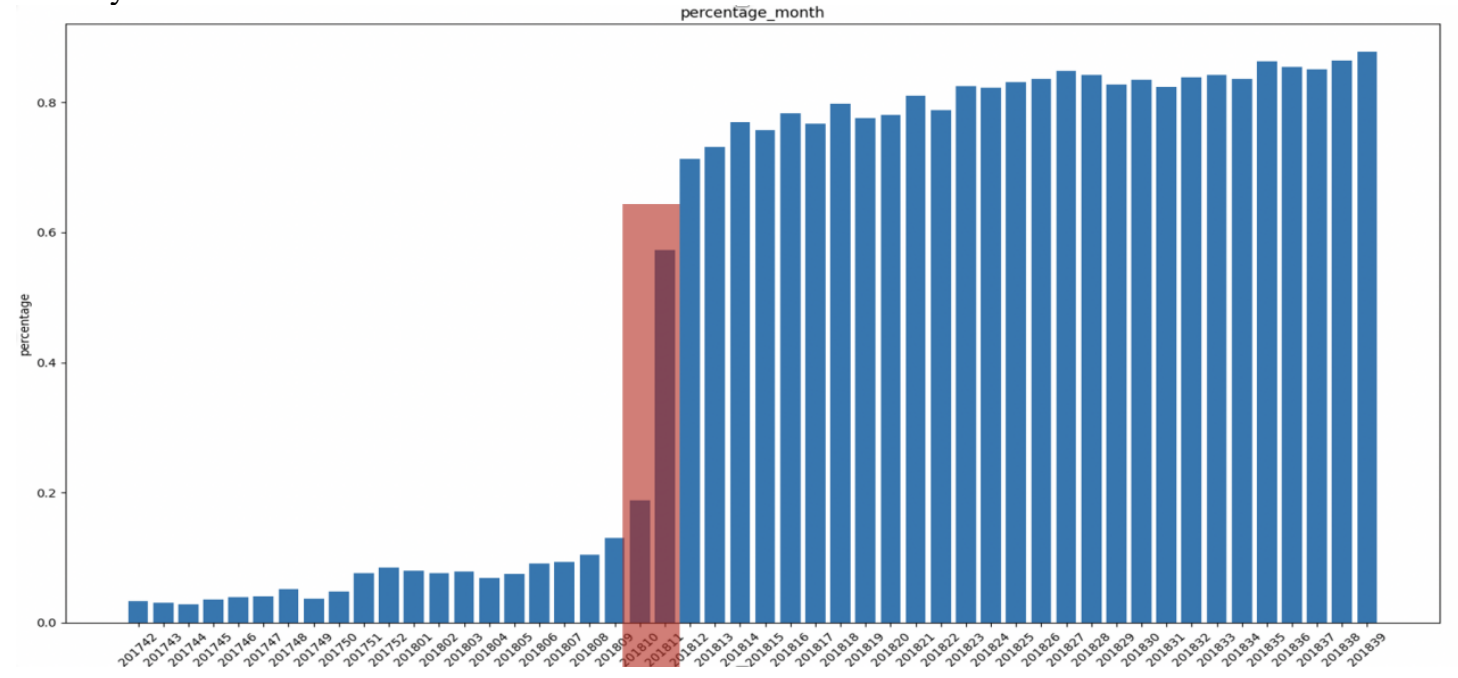

Figure 1. Percentage of articles liked each week

In the author-level analysis, we collect all articles published in SA. The information on each article includes article ID, article body, publication date, rating, and ticker. In this study, we only focus on active existing authors who published articles before and within our study period, which leads to a sample of 189 authors. Because the author's publishing frequency is relatively low, we set two weeks as one time period to build the panel data. Therefore, we obtain 1,512 observations.

In the reader-level analysis, we collect each reader's historical comment information. The information for comments includes user ID, comment ID, comment body, and comment time. We process data similar to that in the author-level analysis. Finally, we obtain a sample of 45,256 observations for 5,657 readers.

\subsection{Variable Measures and Models Development}

3.2.1. Author-level Variable Measures and Models Development. According to the literature, content valence(emotions and tonality) and content volume determine the effectiveness of online content [27]. Content with accuracy, analysis, information, status, and value can generate more praise and comments [28]. According to the hypotheses, we develop our research models for emotional tonality and content volume.

Words used in daily language can reveal individuals' thoughts, emotions, personalities and motivations [29]. The superficial features of articles can be quantified by natural language processing. For example, the frequency of negative words used in this paper captures the keynote of the report, which has been confirmed by prior studies [2,30]. In this study, following the literature, we use Linguistic Inquiry and Word Count (LIWC) to measure the negative emotion and cognitive processes based on the percentage of words in articles. LIWC is a text analysis tool created by using language query and word counting. It compares each English word with a file with 72 dimensions and more than 2,000 words, and outputs the percentage of such words in the total vocabulary of the text [31]. 
LIWC is often used as a tool for quantitative text analysis.

We use the number of articles published by author $i$ within period $t$ (ArticleNum) to measure content volume. At the same time, the author's writing experience will also affect the author's writing frequency and writing style. For example, the author's proficiency will increase the publishing frequency. We choose Age to show the author's experience as the control variable.

The selection and definitions of author-level variables are shown in Table 1.

Table 1. Measurement of variables in author-level

\begin{tabular}{c|c|l}
\hline Variable type & Variable & \multicolumn{1}{c}{ Measure } \\
\hline Independent Variable & After & $\begin{array}{l}\text { Dummy variable indicates whether period } t \text { is after the } \\
\text { event. }\end{array}$ \\
\hline \multirow{2}{*}{ Dependent Variable } & ArticleNum & The number of articles published by author $i$ in period $t$. \\
\cline { 2 - 3 } & NegEmo & $\begin{array}{l}\text { The percentage of negative words generated by author } \\
i \text { in period } t .\end{array}$ \\
\cline { 2 - 3 } & CogProc & $\begin{array}{l}\text { The percentage of words shows cognitive processes } \\
\text { generated by author } i \text { in period } t .\end{array}$ \\
\hline Control Variable & $\log ($ Age +1$)$ & $\begin{array}{l}\text { The number of articles published by author } i \text { before } \\
\text { period } t .\end{array}$ \\
\hline
\end{tabular}

To investigate the effects of the "likes" function on the author's generated-content, we develop the following regression models according to H1a (H1b), $\mathrm{H} 2$, and $\mathrm{H} 3$ :

$$
\begin{aligned}
& \text { ArticleNum }=\beta_{0}+\beta_{1} \text { After }+\beta_{2} \log (\text { Age }+1) \\
& \text { NegEmo }=\beta_{0}+\beta_{1} \text { After }+\beta_{2} \log (\text { Age }+1) \\
& \text { CogProc }=\beta_{0}+\beta_{1} \text { after }+\beta_{2} \log (\text { Age }+1)
\end{aligned}
$$

\subsubsection{Reader-level Variable Measures and Model} Development. We also need to measure the emotional tonality and content volume of readers' comments. Based on the reader-level hypotheses, we still use the quantitative results of LIWC to measure the proportion of negative emotions, and use the number of comments published within two weeks as the measurement of publishing frequency. Readers' experience in writing comments may also affect their emotional tonality and publishing frequency, so we control for the number of weeks readers write reviews. All the variables at the reader-level are summarized in Table 2.

To investigate the effects of the "likes" function on the reader's generated-content, we build the following regression models according to $\mathrm{H} 4$ and $\mathrm{H} 5 \mathrm{a}(\mathrm{H} 5 \mathrm{~b})$ :

$$
\begin{aligned}
& \text { NegEmo }=\beta_{0}+\beta_{1} \text { After }+\beta_{2} \log (\text { Age }+1) \\
& \text { CommentNum }=\beta_{0}+\beta_{1} \text { After }+\beta_{2} \log (\text { Age }+1)
\end{aligned}
$$

Table 2. Measurement of variables in reader-level

\begin{tabular}{c|c|l}
\hline Variable type & Variable & \multicolumn{1}{c}{ Measure } \\
\hline Independent Variable & After & Dummy variable indicates whether period t is after the event. \\
\hline \multirow{2}{*}{ Dependent Variable } & CommentNum & The number of articles published by reader i in period t. \\
\cline { 2 - 3 } & NegEmo & $\begin{array}{l}\text { The percentage of negative words generated by author i in } \\
\text { period t. }\end{array}$ \\
\hline Control Variable & $\log ($ Age +1$)$ & $\begin{array}{l}\text { The number of comments published by reader i before } \\
\text { period t. }\end{array}$ \\
\hline
\end{tabular}

\section{Empirical results}

\subsection{Descriptive Analyses}

Table 3 reports the descriptive statistical results of the authors and their articles. In the 8-week time window, authors published 0.53 articles every two weeks on average, the average output is relatively small; the average length of articles is 1,736 English words, with 13,270 words at most, and the text volume is quite high. However, the minimum length of the article is 2 words, because some authors only show the trend chart and analysis table in the article. The proportion of negative emotion words is relatively small. The average writing experience of the authors in the sample is relatively rich. The standard deviation is relatively large, which indicates that there is a large difference in the author's writing experience, so it is necessary to control the author's writing experience. 
Table 3. Descriptive statistics at the author-level

\begin{tabular}{c|ccccc}
\hline Variable & Obs\# & Mean & Std. Dev. & Min & Max \\
\hline ArticleNum & 1,512 & 0.536 & 0.824 & 0 & 6 \\
ArticleLength & 681 & $1,736.528$ & $1,216.436$ & 2 & 13,270 \\
CogProc & 681 & 9.591 & 2.198 & 0 & 33.330 \\
NegEmo & 681 & 1.064 & 0.536 & 0 & 3.610 \\
Log(Age+1) & 1,512 & 166.402 & 152.742 & 0 & 613.500 \\
\hline
\end{tabular}

Table 4. Descriptive statistics at reader-level

\begin{tabular}{c|ccccc}
\hline Variable & Obs\# & Mean & Std. Dev. & Min & Max \\
\hline CommentNum & 45,256 & 0.689 & 1.672 & 0 & 73.5 \\
CommentLength & 22,168 & 45.466 & 54.559 & 1 & 1,357 \\
NegEmo & 22,168 & 1.627 & 3.429 & 0 & 100 \\
Log(Age+1) & 45,256 & 6.708 & 5.361 & 0 & 16.5 \\
\hline
\end{tabular}

According to the results in Table 4, we can observe that readers do not seem to be very active in writing comments. On average, 0.68 comments are generated every two weeks, and the standard error is large. The standard error of the length of comments also shows the same trend, the standard deviation is relatively large, the shortest comment has only one word, while the longest comment has 1,357 words. The proportion of negative emotion words is relatively balanced, with an average of $1.6 \%$ of negative emotion tendency. Compared with the descriptive statistics of the author's data, it seems that readers' experience in writing comments is not particularly sufficient. The average experience is 6.7 weeks, and the most experienced is only 16.5 weeks. A considerable number of new people or "tourists" leave comments after browsing the articles.

\subsection{Regression Results}

4.2.1. Regression Results for Author-level Analyses. In order to test $\mathrm{H} 1 \mathrm{a}, \mathrm{H} 1 \mathrm{~b}, \mathrm{H} 2$, and $\mathrm{H} 3$, we estimate models (1), (2), (3), and the regression results are shown in Table 5. After the launching of the "likes" function, the author significantly increases the frequency of publishing articles, and shows more negative emotions and a higher proportion of reasoning analyses. These results support $\mathrm{H} 1 \mathrm{a}, \mathrm{H} 2$ and $\mathrm{H} 3$.

Table 5. Regression results of author-level

\begin{tabular}{cccc}
\hline & (1) ArticleNum & (2) NegEmo & (3) CogProc \\
\hline after & $-0.150^{\star \star \star}$ & $0.073^{\star}$ & $0.376^{\star \star *}$ \\
& $(0.039)$ & $(0.041)$ & $(0.122)$ \\
Log(Age+1) & & & -0.036 \\
& 0.014 & -0.069 & $(0.163)$ \\
& $(0.046)$ & $(0.088)$ & \\
Constant & & & $9.578^{\star \star \star}$ \\
& $0.549^{\star \star \star}$ & $1.342^{\star \star \star}$ & $(0.704)$ \\
\hline Obs\# & $(0.196)$ & $(0.387)$ & 681 \\
R2 & 1,512 & 681 & 0.014 \\
\hline
\end{tabular}

Standard errors are reported in parentheses ${ }^{*} p<0.10,{ }^{* *} p<0.05,{ }^{* * *} p<0.01$

After introducing the new function, the author has a new opportunity to get approval feedback, and there is another way to attract readers' attention to further improve their psychological encouragement and get satisfaction. Therefore, "catering" to readers' preferences and attracting their attention will become 
a goal of the author. The specific performance is that the author spends more time polishing his works, and integrates more analytical reasoning and negative emotions to attract the attention of readers, and makes certain changes in order to obtain more likes.

4.2.2. Regression Results for Reader-level Analyses. Table 6 shows the empirical results of the empirical Models (4) and (5), and tests the changes in the frequency of readers' comments and the negative emotions of the content. The result of Model (4) shows that readers do not give up writing comments because of the convenient "likes" button, but increase the frequency of writing comments; the proportion of negative words has no significant change. These results support our hypotheses 4 and 5b. We also know that likes do not cover the comments that show agreement, but promote the generation of comments.

Table 6. Regression results of reader-level

\begin{tabular}{ccc}
\hline & $(4)$ NegEmo & (5) CommentNum \\
\hline After & -0.044 & $0.083^{\star \star \star}$ \\
& $(0.095)$ & $(0.026)$ \\
Log(Age+1) & $0.108^{\star \star}$ & $-0.094^{\star \star \star}$ \\
& $(0.046)$ & $(0.016)$ \\
Constant & & $0.804^{\star \star *}$ \\
& $1.468^{\star \star \star}$ & $(0.019)$ \\
\hline Obs\# & $(0.043)$ & 45,256 \\
\hline
\end{tabular}

Standard errors are reported in parentheses

${ }^{*} p<0.10,{ }^{* *} p<0.05,{ }^{* * *} p<0.01$

\section{Robustness Checks}

Table 7. Robust test of different time windows at author-level

\begin{tabular}{|c|c|c|c|c|c|c|}
\hline & \multicolumn{2}{|c|}{ (1) ArticleNum } & \multicolumn{2}{|c|}{ (2) NegEmo } & \multicolumn{2}{|c|}{ (3) CogProc } \\
\hline & 6-weeks & 10-weeks & 6-weeks & 10-weeks & 6-weeks & 10-weeks \\
\hline \multirow[t]{2}{*}{ After } & $-0.174^{\star \star \star}$ & $-0.084^{\star \star}$ & $0.082^{\star}$ & $0.066^{\star}$ & $0.469^{\star \star}$ & $0.287^{\star \star \star}$ \\
\hline & $(0.055)$ & $(0.033)$ & $(0.046)$ & $(0.037)$ & $(0.191)$ & $(0.104)$ \\
\hline \multirow[t]{2}{*}{$\log (A g e+1)$} & 0.034 & 0.020 & -0.024 & -0.079 & -0.224 & 0.017 \\
\hline & $(0.074)$ & $(0.037)$ & $(0.110)$ & $(0.065)$ & $(0.251)$ & $(0.127)$ \\
\hline \multirow[t]{2}{*}{ Constant } & $0.560^{\star}$ & $0.422^{\star \star \star}$ & $1.124^{\star \star}$ & $1.393^{\star \star \star}$ & $10.400^{\star \star \star}$ & $9.439^{\star \star \star}$ \\
\hline & $(0.312)$ & $(0.158)$ & (0.482) & $(0.286)$ & (1.077) & $(0.554)$ \\
\hline Authors & 146 & 230 & 146 & 230 & 146 & 230 \\
\hline Obs\# & 876 & 2,300 & 466 & 920 & 466 & 920 \\
\hline $\mathrm{R}^{2}$ & 0.016 & 0.004 & 0.012 & 0.005 & 0.024 & 0.009 \\
\hline
\end{tabular}

Standard errors are reported in parentheses

${ }^{*} p<0.10,{ }^{* *} p<0.05,{ }^{* * *} p<0.01$

In natural experiment research, the selection of time window may have a certain impact on the results. For example, in a longer time window, even the impact of a major event will be diluted by other events with the passage of time; in a shorter time window, the event may not show a certain impact in a short time window because the public may do not form cognition or the lag of event impact. So, the results of this study may only 
appear in a specific time window, the results are not robust and accidental, so we have some concerns about the time window. In this part, we tried 6-week and 10week windows respectively to test the Models (1) (5) in the same way. The regression results are shown in Tables 7 and 8 .
According to the results of Tables 7 and 8, our conclusion is significant in the 6-week time window and the 10-week time window. Suppose that H1a, H2, H3, $\mathrm{H} 4$, and $\mathrm{H} 5 \mathrm{~b}$ are still confirmed. It shows that the findings of this study are robust.

Table 8. Robust test of different time windows at reader-level

\begin{tabular}{|c|c|c|c|c|}
\hline & \multicolumn{2}{|c|}{ (4) NegEmo } & \multicolumn{2}{|c|}{ (5) CommentNum } \\
\hline & 6-weeks & 10-weeks & 6-weeks & 10-weeks \\
\hline \multirow[t]{2}{*}{ After } & -0.047 & -0.080 & $0.274^{\star \star \star}$ & -0.080 \\
\hline & $(0.130)$ & $(0.081)$ & $(0.036)$ & $(0.081)$ \\
\hline \multirow[t]{2}{*}{$\log (A g e+1)$} & 0.101 & $0.109^{\star \star \star}$ & $-0.236^{\star * *}$ & $0.109^{\star \star \star}$ \\
\hline & $(0.074)$ & $(0.037)$ & $(0.021)$ & $(0.037)$ \\
\hline \multirow[t]{2}{*}{ Constant } & $1.519^{\star \star \star}$ & $1.437^{\star \star \star}$ & $0.987^{\star \star \star}$ & $1.437^{\star \star \star}$ \\
\hline & $(0.060)$ & $(0.039)$ & $(0.021)$ & $(0.039)$ \\
\hline Readers & 4,422 & 65,551 & 4,422 & 6,551 \\
\hline Obs\# & 14,811 & 29,051 & 26,532 & 65,510 \\
\hline $\mathrm{R}^{2}$ & 0.001 & 0.001 & 0.007 & 0.001 \\
\hline
\end{tabular}

Standard errors are reported in parentheses

${ }^{*} p<0.10,{ }^{* *} p<0.05,{ }^{* * *} p<0.01$

\section{Conclusions}

As a simple feedback mechanism, "likes" brings great psychological incentive to authors. As another kind of feedback, comments need the commentators to organize language to express approval, neutrality, opposition, or supplement, which causes a higher information cost. The new "likes" function of Seeking Alpha in 2018 provides the opportunity to conduct this research.

From the author's point of view, getting attention, response and psychological encouragement is the main source of strength for authors. After the "likes" function launched, a higher proportion of cognitive vocabulary and fewer articles were published by authors, which indicates that authors show more reasoning and rational thinking, and spend more time contributing each article. Meanwhile, the article's negative emotions also have a more significant improvement. This study speculates that such articles will attract more investors who aims to obtain negative information disclosure and avoid investment risks.

From the reader's point of view, because readers seek investment advice to reduce their information asymmetry, they absorb the analyses and opinions of other investors with lower information processing costs. After the "likes" function was launched, readers make more comments and the negative emotion of comments does not decrease significantly, indicating that "likes" does not replace comments, and on the contrary, as a new function with low processing cost, "likes" brings higher user participation and reader response.

Theoretically, our study enriches the existing literature on feedback mechanisms, and explores the relationship between "likes" and "comments". The findings show that "likes" is not a substitute for "comments", and on the contrary, it improves the overall user participation. In reality, this study provides suggestions for OICs authors to attract more attention, and provides implications for the OIC managers to build a positive community and maintain vitality. At the same time, there are some limitations in this study, which will be addressed in the future work. First, there is no specific mechanism to explore how "likes" improves the participation of comments. Besides, we do not subdivide comments from commentators and replies from authors.

\section{References}

[1] SEC. 2012. "Investment Adviser Use of Social Media," 7, pp. 21-22.

[2] Chen, H., De, P., Hu, Y. J., and Hwang, B.-H. 2014. "Wisdom of Crowds: The Value of Stock Opinions Transmitted through Social Media," The Review of Financial Studies (27:5), pp. 1367-1403.

[3] Gomez, E., Heflin, F., Moon, J., and Warren, J. 2018. "Crowdsourced Financial Analysis and Information 
Asymmetry at Earnings Announcements," Georgia Tech Scheller College of Business Research Paper), pp. 18-45.

[4] Campbell, J. L., DeAngelis, M. D., and Moon, J. R. 2019. "Skin in the Game: Personal Stock Holdings and Investors' Response to Stock Analysis on Social Media," Review of Accounting Studies (24:3), pp. 731-779.

[5] Ren, Y., Drenner, S., and Terveen, L. 2012. "Bulding Member Attachment in Online Communities: Applying Theories of Group Identity and Interpersonal Bonds," MIS Quarterly (36:3), pp. 841-864.

[6] Ding, R., Zhou, H., and Li, Y. 2019. "Social Media, Financial Reporting Opacity, and Return Comovement: Evidence from Seeking Alpha," Journal of Financial Markets (50), pp. 1-25.

[7] Fu, P.-W., Wu, C.-C., and Cho, Y.-J. 2017. "What Makes Users Share Content on Facebook? Compatibility among Psychological Incentive, Social Capital Focus, and Content Type," Computers in Human Behavior (67), pp. 23-32.

[8] Zhang, X. M., and Zhu, F. 2011. "Group Size and Incentives to Contribute: A Natural Experiment at Chinese Wikipedia," American Economic Review (101:4), pp. 1601-1615.

[9] Ding, C., Cheng, H. K., Duan, Y., and Jin, Y. 2017. "The Power of the "Like" Button: The Impact of Social Media on Box Office," Decision Support Systems (94), pp. 77-84.

[10] Sumner, E. M., Ruge-Jones, L., and Alcorn, D. 2018. "A Functional Approach to the Facebook Like Button: An Exploration of Meaning, Interpersonal Functionality, and Potential Alternative Response Buttons," New Media \& Society (20:4), pp. 14511469.

[11] Andreoni, J. 1989. "Giving with Impure Altruism: Applications to Charity and Ricardian Equivalence," Journal of Political Economy (97:6), pp. 1447-1458.

[12] Bishop, J. 2007. "Increasing Participation in Online Communities: A Framework for Human-Computer Interaction," Computers in Human Behavior (23:4), pp. 1881-1893.

[13] Yu, Y., Khern-am-nuai, W., and Pinsonneault, A. 2018. "The Impact of Performance-Contingent Monetary Incentives on User-Generated Content Contribution," Americas' Conference on Information Systems, New Orleans.

[14] Chen, H., Hu, Y. J., and Huang, S. 2019. "Monetary Incentive and Stock Opinions on Social Media," Journal of Management Information Systems (36:2), pp. 391-417.

[15] Bhattacharyya, S., Banerjee, S., Bose, I., and Kankanhalli, A. 2020. "Temporal Effects of Repeated Recognition and Lack of Recognition on Online Community Contributions," Journal of Management Information Systems (37:2), pp. 536562.

[16] Bretschneider, U., Rajagopalan, B., and Leimeister, J. M. 2012. "Idea Generation in Virtual
Communities for Innovation: The Influence of Participantss Motivation on Idea Quality," in: Ssrn Electronic Journal.

[17] Kahneman, D., Slovic, P., and Tversky, A. 1978. "Judgment under Uncertainty: Heuristics and Biases," Uncertainty in Economics (34:3), pp. 17-34.

[18] Peeters, G., and Czapinski, J. 1990. "PositiveNegative Asymmetry in Evaluations: The Distinction between Affective and Informational Negativity Effects," European Review of Social Psychology (1:1), pp. 33-60.

[19] Ito, T. A., Larsen, J. T., Smith, N. K., and Cacioppo, J. T. 1998. "Negative Information Weighs More Heavily on the Brain: The Negativity Bias in Evaluative Categorizations," Journal of Personality \& Social Psychology (75:4), pp. 887-900.

[20] Simon, H. A. 1978. "Rationality as Process and as Product of Thought," The American economic review (68:2), pp. 1-16.

[21] Tuttle, B., and Burton, F. G. 1999. "The Effects of a Modest Incentive on Information Overload in an Investment Analysis Task," Accounting, Organizations and Society (24:8), pp. 673-687.

[22] Barber, B. M., and Odean, T. 2013. "The Behavior of Individual Investors," in Handbook of the Economics of Finance. pp. 1533-1570.

[23] Kothari, S. P., So, E., and Verdi, R. 2016. "Analysts' Forecasts and Asset Pricing: A Survey," Annual Review of Financial Economics (8:1), pp. 197-219.

[24] Rosen, L. D. 2012. "The Power of "Like"." Retrieved 6-15, 2012, from https://www.psychologytoday.com/intl/blog/rewire d-the-psychology-technology/201207/the-power

[25] Wellman, B., Quan-Haase, A., Boase, J., Chen, W., Hampton, K., Díaz, I., and Miyata, K. 2003. "The Social Affordances of the Internet for Networked Individualism," Journal of Computer-Mediated Communication (8:3), pp. 1-24.

[26] Bagozzi, R. P., and Dholakia, U. M. 2006. "Antecedents and Purchase Consequences of Customer Participation in Small Group Brand Communities," International Journal of Research in Marketing (23:1), pp. 45-61.

[27] Peters, K., Chen, Y., Kaplan, A. M., Ognibeni, B., and Pauwels, K. 2013. "Social Media Metrics - a Framework and Guidelines for Managing Social Media," Journal of Interactive Marketing (27:4), pp. 281-298.

[28] Moe, W. W., and Trusov, M. 2011. "The Value of Social Dynamics in Online Product Ratings Forums," Journal of Marketing Research (48:3), pp. 444-456.

[29] Graesser, A. C., Lu, S., Jackson, G. T., Mitchell, H. H., Ventura, M., Olney, A., and Louwerse, M. M. 2004. "Autotutor: A Tutor with Dialogue in Natural Language," Behavior Research Methods, Instruments \& Computers (36:2), pp. 180-192.

[30] Das, S. R., and Chen, M. Y. 2007. "Yahoo! For Amazon: Sentiment Extraction from Small Talk on the Web," Management Science (53:9), pp. 13751388 . 
[31] Pennebaker, J. W., Boyd, R. L., Jordan, K., and Blackburn, K. 2015. "The Development and
Psychometric Properties of Liwc2015," University of Texas at Austin, Austin, TX. 